\title{
Monitoring Genetic and Metabolic Potential for In Situ Bioremediation: Mass Spectrometry
}

Oak Ridge National Laboratory

September 1997

\section{Progress Report}

\section{Principal Investigator}

Michelle V. Buchanan

(423) 574-4868 (Phone)

(423) 576-8559 (Fax)

buchananmv@ornl.gov

Oak Ridge National Laboratory

Oak Ridge, TN 37831-6365

\section{Co-Investigators}

Gregory B. Hurst

(423) 574-7469 (Phone)

(423) 576-8559 (Fax)

h9g@ornl.gov

Oak Ridge National Laboratory

Oak Ridge, TN 37831-6365

Scott A. McLuckey

(423) 574-2848 (Phone)

(423) 576-8559 (Fax)

luy@ornl.gov

Oak Ridge National Laboratory

Oak Ridge, TN 37831-6365

Phillip F. Britt

(423) 574-5029 (Phone)

(423) 576-5235 (Fax)

bfp@ornl.gov

Oak Ridge National Laboratory

Oak Ridge, TN 37831-6365

Mitchel J. Doktycz

(423) 574-6204 (Phone)

(423) 574-6210 (Fax)

okz@ornl.gov

Oak Ridge National Laboratory

Oak Ridge, TN 37831-6365

\section{Contributors}

Mary Lidstrom, University of Washington

Andria Costello, University of Washington

Kristal Weaver, Oak Ridge Institute for Science and Education 


\section{Research Objective}

A number of U.S. Department of Energy (DOE) sites are contaminated with mixtures of dense non-aqueous phase liquids (DNAPLs) such as carbon tetrachloride, chloroform,. perchloroethylene, and trichloroethylene. At many of these sites, in situ microbial bioremediation is an attractive strategy for cleanup because it has the potential to degrade DNAPLs in situ without producing toxic byproducts. A rapid screening method to determine the broad range metabolic and genetic potential for contaminant degradation would greatly reduce the cost and time involved in assessment for in situ bioremediation as well as for monitoring ongoing bioremediation treatment.

In this project, the ORNL Organic Mass Spectrometry (OMS) group is developing massspectrometry-based methods to screen for the genetic and metabolic potential for assessment and monitoring of in situ bioremediation of DNAPLs. In close collaboration, Professor Mary Lidstrom's group at the University of Washington is identifying short DNA sequences related to microbial processes involved in the biodegradation of pollutants. This work will lay the foundation for development of a field-portable mass-spectrometry-based technique for rapid assessment and monitoring of bioremediation processes on site.

\section{Research Statement}

DNAPLs, including carbon tetrachloride, chloroform, perchloroethylene, and trichloroethylene, constitute the largest class of organic contaminants at DOE facilities. In situ bioremediation of DNAPLs is an attractive possibility for cleanup of contaminated aquifers because it has the potential to minimize time, cost, and environmental disruption.

To develop bioremediation into a reliable and cost-effective treatment strategy for cleanup of DNAPLs, it is necessary to have available a rapid screening tool for assessing the metabolic and genetic potential of the indigenous microbial population for destroying pollutants. Clearly, the development of novel bioremediation technologies, informed decision-making regarding bioremediation as a treatment option, and the optimization of a cleanup system once implemented will require in-depth information on the bacteria present at each site and the processes they can carry out. Currently such information is generated by labor- and timeintensive treatability tests in the laboratory, and these do not generally assess a broad range of metabolic processes.

The explosion of information in the DNA sequence database raises the possibility of developing diagnostic DNA signatures for key microbial processes. It is already feasible to use molecular techniques to screen for a number of functional types of bacteria that play a role in biodegradation: methanogens, sulfate-reducing bacteria, denitrifiers, nitrifiers, methanotrophs, and bacteria containing broad-spectrum iron and flavo-oxygenases. Exploiting these diagnostic DNA sequences would require a rapid analytical method for identifying specific signatures. Current methods involve amplifying DNA using the polymerase chain reaction (PCR) to make multiple copies of a characteristic DNA sequence. Subsequent analysis and detection of PCR products is conventionally performed by gel electrophoresis or hybridization assays, requiring several hours or more to perform. Matrix-assisted laser desorption/ionization mass spectrometry (MALDI-MS) is emerging as a technology for detection of PCR products, yielding information similar to electrophoresis, but with analysis times reduced by several orders of magnitude. Mass 
spectrometry also has the potential to be automated, incorporating sample preparation and detection. In many cases, these DNA signatures would be direct indicators of metabolic potential.

The goal of this work is a more rapid analysis of microbial PCR products using MALDI-MS instead of traditional gel electrophoretic methods. The long-term payoff of the described research would be a much higher-throughput analytical method with enhanced accuracy, providing a valuable tool for microbiologists in evaluating and modifying environmental microbial populations. Ultimately, this work should lead to the identification of a suite of diagnostic DNA sequences that can be measured to assess bioremediation processes. Although construction of such a device is not included in the scope of the present work, the technologies under development could be incorporated into a field-portable mass spectrometer for on-site evaluation of factors relevant to the use of bioremediation.

\section{Research Progress}

\section{Improved Instrumentation Capabilities at ORNL}

A number of improvements have been implemented for MALDI-MS detection of PCR products. These include an upgrade of our existing in-house-constructed time-of-flight (TOF) mass spectrometer and purchase of a commercial mass spectrometer.

The existing MALDI-TOF instrument, which previously had limited capabilities for delayed extraction, was upgraded to allow higher-voltage pulsing, with resulting improvements in the accessible mass range and resolution. Previously, the high-voltage ion-extraction pulse was limited to 400 volts, with a total ion accelerating potential of about 5000 volts. Under these conditions, small oligonucleotides (up to 30 mers) could be detected with good resolution, but larger species could not be detected because of the low total accelerating potential.

Conversely, with continuous (not delayed) ion extraction, it was possible to apply larger accelerating potentials and thereby detect oligonucleotides containing up to 168 bases, but resolution was poor. When fully implemented, the new electronics will allow us to pulse 6,000 volts, with a total accelerating potential of up to approximately 20,000 volts. These improvements will increase both the observed sensitivity and resolution. Using an intermediate implementation of the upgraded pulsing electronics for delayed extraction, we observed singlebase resolution for a mix of synthetic oligonucleotides containing 40 to 60 adenosine units (A-), as Fig. 1 shows. Before this upgrade, the MALDI-MS spectrum of this mixture was a broad, unresolved hump.

In addition to upgrades to the existing TOF, the OMS group at ORNL has acquired a PerSeptive Biosystems MALDI-TOF mass spectrometer that has expanded our ability to develop analyses of PCR products obtained from bacteria that are relevant to bioremediation efforts. The instrument incorporates a 100-well sample plate and an automated data system that greatly increases throughput, allowing more samples to be analyzed, more rapid evaluation of sample preparation procedures, optimization of PCR parameters, and examination of new methods for combining the DNA with the MALDI matrix. 


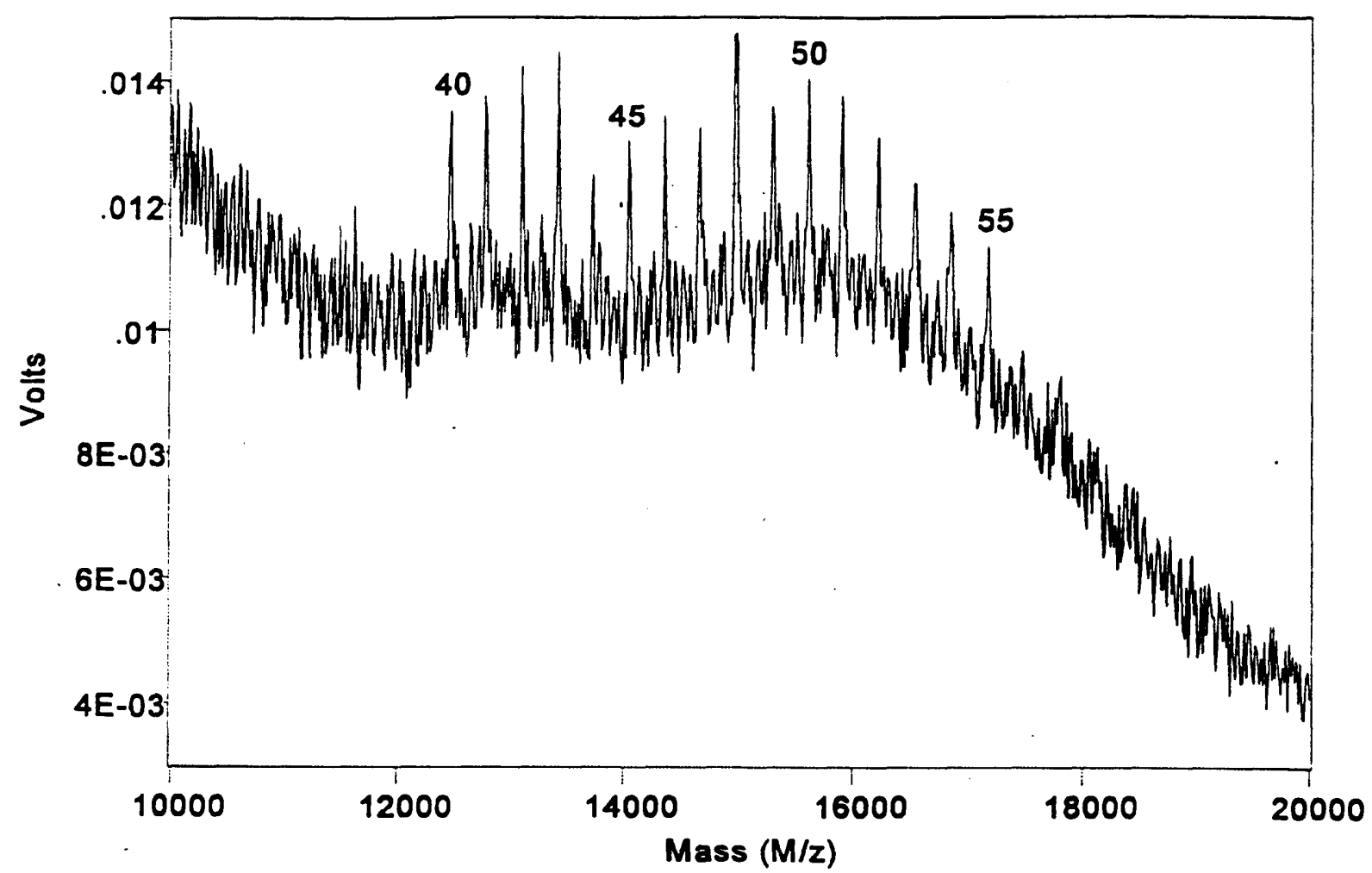

Figure 1. MALDI-TOF spectrum of a poly-A oligonucleotide mixture, $A_{40}-A_{60}$, obtained using the upgraded ORNL-built mass spectrometer.

\section{Development and Detection of PCR Products for MALDI-MS}

Currently, MALDI-MS is capable of detecting oligonucleotides up to about 60 bases long with better than single-base resolution, as demonstrated in Fig. 1. While OMS is working to extend this capability, it is of immediate importance to develop PCR products in this size range. Professor Lidstrom's group designed a model system for interfacing MALDI-MS with PCR amplification based on the gene for $p m o A$, the active site subunit of particulate methane monooxygenase, which is a bacterial enzyme that can oxidize trichloroethylene. PCR primer pairs were designed to amplify relatively short segments of this gene in two bacterial species-a Type I methanotroph (Methylomicrobium albus, strain BG8) and a Type II methanotroph (Methylosinus trichosporium, strain OB3b). The sequences for these two PCR assays were deduced from a large number of $p m o A$ sequences and are designed to pick up all $p m o A$ genes, but not the closest relative, which is a gene encoding the analogous subunit of the ammonia monooxygenase.

The PCR product for the Type I methanotroph is 99 bases in length, while that from the Type II methanotroph is 56 bases long. Using these primer sets, genomic DNA from these two species, and advice on initial amplification conditions supplied by Prof. Lidstrom, both of these PCR amplifications were performed at ORNL using a PCR machine purchased with EMSP funds. 
Following generation of the Type I and Type II PCR products, we evaluated a number of rapid separation strategies for removing components of the amplification reaction, such as salts and buffers, that our previous work has shown to interfere with the MALDI-MS analysis. These separations included membrane dialysis, centrifuge-based molecular weight cutoff filters, reverse-phase adsorption, and silica-based ion exchange. Reverse-phase high-performance liquid chromatography analysis allowed a semi-quantitative measure of the recovery of PCR product for each type of separation.

Subsequent examination of the purified products by MALDI-MS revealed that for most of the separation techniques evaluate\& either removal of contaminants was not satisfactory or recovery of the PCR product was unacceptably low. However, the reverse-phase separation using a commercially-available cartridge 'format appears promising. The manufacturer's desalting protocol for these cartridges, which we are presently modifying to suit our needs, is fairly rapid and could be automated and perhaps adapted to a 96-well microtiter plate format.

Figure 2 shows MAIDI mass spectra of the Type I and Type II short PCR products generated using Prof. Lidstrom's model system. The main peak observed for the Type II product, 56 bases in length, is about 20 daltons wide at half maximum intensity. Single-base differences in length (about 300 Daltons) could easily be distinguished in this mass range. (Under favorable conditions, even base substitutions could be distinguished with this resolution. For instance, substitution of a $\mathrm{C}$ for a $\mathrm{G}$ results in a 40-dalton mass difference.) The Type I product, 99 bases in length, is substantially broader-approximately 650 daltons, or about two nucleotide units.

The spectrum in Fig. 2 for the 56 mer (Type I) was obtained using a small aliquot from a single 100-microliter PCR reaction, while the 99 mer (Type II) spectrum required pooling of several PCR reactions. Subsequent results indicate that the 99 mer can also be detected from a single PCR reaction. This level of sensitivity indicates that MALDI-MS has considerable potential to compete with existing methods for detecting PCR products in this size range.

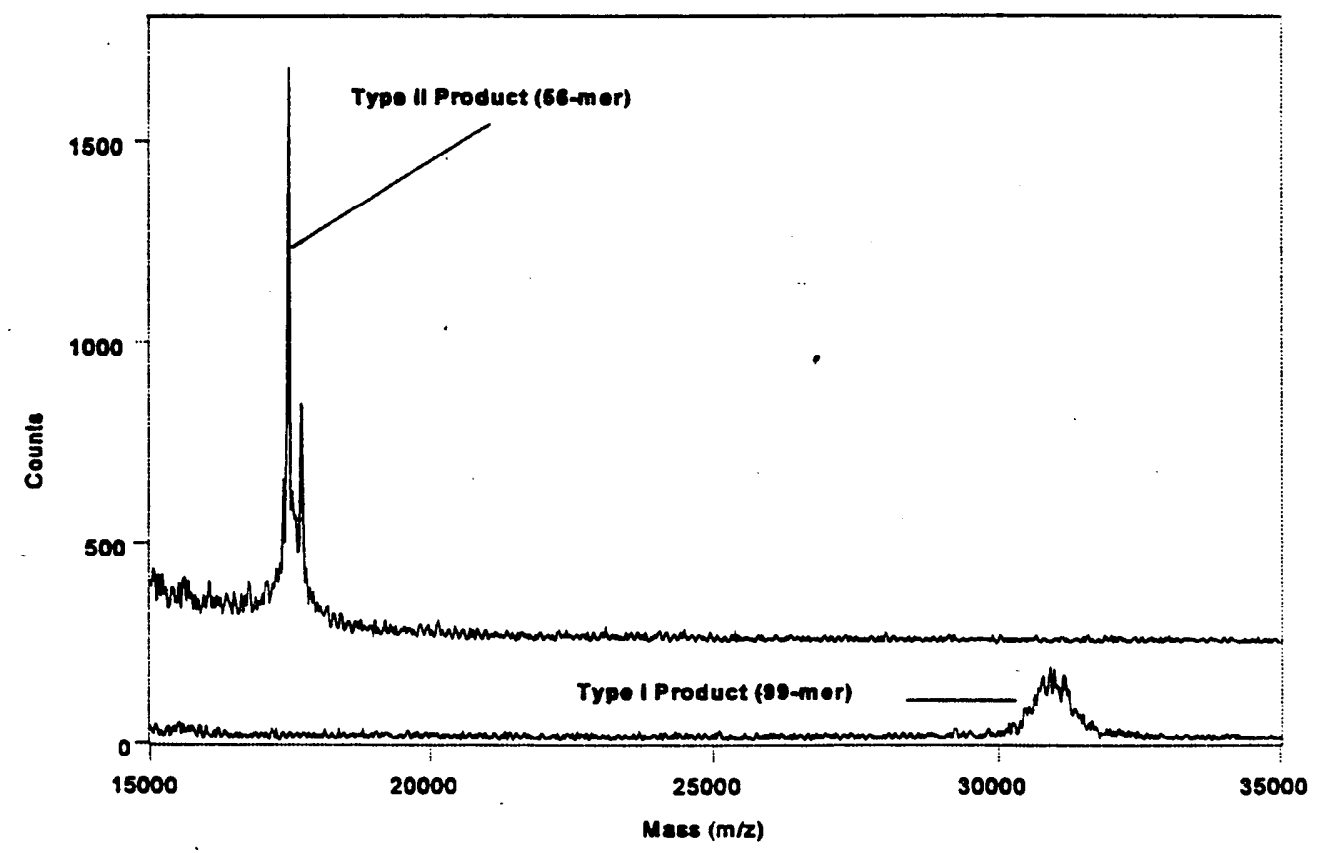

Figure 2. MALDI-TOF mass spectra of two short PCR products from different methanotrophic bacteria. 
In addition to the short PCR assays and genomic DNA mentioned previously, Prof. Lidstrom's group also supplied primer sequences currently in use in her laboratory for amplification of roughly a 500-base segment of the particulate methane monooxygenase gene. This product will be useful in upcoming MALDI-MS experiments, such as development of protocols for MALDI-MS of larger PCR products and investigation of hybridization studies using MALDI-MS detection of probes.

It should be noted that along with the proposal that led to the current work, Prof. Lidstrom submitted a companion proposal that was not funded. Because her laboratory's contribution is critical to the success of this project, we have arranged to support a graduate student in her laboratory through a subcontract arrangement, using funds from our EMSP grant.

\section{Summary of Accomplishments}

- Designed and implemented PCR protocols for amplification of short DNA sequences from methane monooxygenase genes of two species of methanotrophic bacteria.

- Demonstrated improved MALDI-MS performance for detection of oligonucleotides and PCR products.

- Upgraded electronics for delayed extraction on existing MALDI-MS.

- Acquired instrumentation (PCR machine, MALDI-TOF) to improve efficiency of experimental procedures.

\section{Papers and Abstracts}

Hurst, Gregory B., Kristal Weaver, and Michelle V. Buchanan. "Improved Mass Spectrometric Resolution for PCR Product Size Measurement." To be presented at the Sixth Department of Energy Contractor and Grantee Workshop of the Human Genome Program, Santa Fe, NM, November 9-13, 1997.

Hurst, G. B., K. Weaver, M. V. Buchanan, and M. J. Doktycz. 1997. "Analysis of PCR Products Using Delayed-extraction MALDI-TOF." In Proceedings of the 45th ASMS Conference on Mass Spectrometry and Allied Topics, Palm Springs, Calif., June 1-5, 1997. 